ISSN: 2581-8341

Volume 04 Issue 06 June 2021

DOI: 10.47191/ijcsrr/V4-i6-13, Impact Factor: 5.825

IJCSRR@ 2021

www.ijcsrr.org

\title{
Comparative Study of Synthesis of Polypyrrole Using Chemical Polymerization Technique and Plasmapolymerization
}

\author{
Dr. Chitte Hemant Keshav \\ Satish Pradhan Dnyanasadhana College, Near Eternity Mall, Thane -400 604
}

\begin{abstract}
Polypyrrole (PPy) was synthesized using the Chemical polymerization technique using Ammonium per sulphate (APS), as an oxidant and Plasma Polymerization technique. The polypyrrole synthesized by the chemical oxidation method was in black amorphous powder form. In Plasma polymerization, thin films were created on a glass plate. Structural and morphological properties of the polymer were studied by FTIR, SEM and. X-ray diffraction. The electrical conductivity of chemically prepared PPy was measured by two probe method and was found to be in the range of $10^{-3}$ to $10^{-2} \mathrm{~S} / \mathrm{cm}$ for chemical oxidative method, while that of Plasma polymerization it was around $10^{-3} \mathrm{~S} / \mathrm{cm}$. Other characterization also shows that Chemical polymerization has an edge over plasma polymerization, but plasma polymerization is more impurity-free than chemical polymerization.
\end{abstract}

KEYWORDS: Electrical Conductivity, Polypyrrole, Plasma, Structure, Thin-Film

\section{INTRODUCTION}

The synthesis of conducting polymers is described in detail in several research articles [1]. Conducting polymers can be synthesized in different ways [2] through chemical polymerization, electrochemical polymerization, photochemical polymerization [3], inclusion polymerization [3], solid-state polymerization [4], plasma polymerization [5], and soluble precursor polymer preparation. Synthesis of conducting polymers is considered to be easy through chemical or electrochemical processes, and their molecular chain structure can be modified conveniently by copolymerization or other structural derivations [6]. PPy received great attention because of its high conductivity and good thermal and environmental stability and ease of synthesis. Its properties can be controlled by substituting among its oxidized and reduced states. Its non-toxic nature makes it convenient to be used in various biomedical applications. [7].That is the reason that various approaches have been reported for synthesizing Polypyrrole according to requirement or application parameters. Many researchers have reported polymerization of Polypyrrole using different oxidants for synthesizing Polypyrrole considering final product requirements. Mostly iron chloride $\left(\mathrm{FeCl}_{3}\right)$ has been used as an oxidant for synthesizing polymer films.[8-12] The use of APS (ammonium per sulphate) as an oxidant focuses on conductivity behaviour.

Although plasma polymerizations have been observed and studied for a long time, the mechanism of deposition is not clear [15]. The mechanism depends on the species present during the deposition, of which the type (e.g., radicals, ions) and amount are important. Mechanisms involving ions [16] and radicals have been described to explain the experimental data [17]. At present, many investigators believe that the polymerization mechanism as proposed by Yasuda et al. is the most reasonable concept. In this stepwise initiation - recombination sequence, radicals are formed upon generation of the discharge (most probably by electron impact). These primary radicals recombine to a species that again is radical initiated and so on. Polymer formation may occur at the surface and/or in the plasma phase $[18,19]$ both of which can be explained by this mechanism. Because of the multiple species present in plasma, many reactions may result in the formation of radicals. For instance, in the DC plasma polymerization of Pyrrole, no effect of the sign of the charge on the electrode was found, showing that a radical growth mechanism should apply [20]. It has been postulated that the growth is initiated by radicals present in the film surface, which are generated by impinging $\mathrm{H}^{+}$ions on the cathode and by electrons on the anode.

In this research synthesize PPy was done by two different methods one by chemical oxidative method and another by plasma polymerization method. Both techniques have their advantages and disadvantages. We tried to compare the characteristics of Polypyrrole produced by both these methods and comment on their use in commercial applications. 


\section{International Journal of Current Science Research and Review}

ISSN: 2581-8341

Volume 04 Issue 06 June 2021

DOI: 10.47191/ijcsrr/V4-i6-13, Impact Factor: 5.825

IJCSRR@ 2021

Www.ijcsrr.org

\section{Preparations and analysis technique:}

Pyrrole (Sisco Research laboratory, 99\% pure) was distilled before use. All other reagents and solvents obtained from SDL were of reagent grade and were used as received. The solution was prepared using distilled water. All reactions were conducted at a temperature of $5^{\circ} \mathrm{C}$ [13-15]. The solution of the oxidizing agent, APS, was prepared using distilled water and was used in the ratio of 1:2.4 (monomer: oxidant) [16].

$1 \mathrm{M}$ Pyrrole solution was prepared using distilled water and then mixed with an oxidizing agent Ammonium per sulphate (APS), slowly under constant stirring for 30 minutes. Then the polymerization was conducted for 4 hours under constant stirring. This preparation was kept unagitated for 24 hours so that PPy powder settled down. The Polypyrrole powder was filtered out under vacuum and washed with distilled water several times to remove any impurities present. The Polypyrrole was dried for 2 days at room temperature.), The polypyrrole obtained was in black amorphous powder form.

The chemical bonding was analyzed using FTIR spectrometer model Perkin-Elmer Ltd. system 2000 using KBr pellets. The Polypyrrole was characterized by XRD using PANalytical (Philips), model XpertPro. The morphology of Polypyrrole was examined using Scanning Electron Microscope (SEM) JEOL make JSM5400 model. The Polypyrrole was tested for conductivity by preparing its pellets of the area of cross-section of $1 \mathrm{sq} \cdot \mathrm{cm}$. and $1 \mathrm{~mm}$. thickness. The conductivity was tested by two probe apparatus fabricated in our laboratory. It consists of a copper plate that serves as one electrode, heated from the bottom to raise the temperature. The second electrode was a copper rod which was spring-loaded to give enough pressure on the sample pallet so that electrical contact is maintained. The conductivity was measured at room temperature $\theta$ in the voltage range from 0 to $12 \mathrm{~V}$.

\section{RESULTS AND CONCLUSIONS:}

\section{FTIR spectroscopy:}

The FTIR spectrum of PPy prepared is represented in Fig. 1. It shows strong absorption bands at around $3435 \mathrm{~cm}^{-1}$ corresponding to N-H stretching. Other medium intensity peaks are observed at around $2926 \mathrm{~cm}^{-1}-2854 \mathrm{~cm}^{-1}$ which can be attributed to aromatic $\mathrm{C}-\mathrm{H}$ stretching vibrations. The absorption band at $1534 \mathrm{~cm}^{-1}$ is related to $\mathrm{C}=\mathrm{C} / \mathrm{C}-\mathrm{C}$ stretching vibrations of the Pyrrole ring. The band at $1471 \mathrm{~cm}^{-1}$ reflects the C-N stretching vibrations of the ring. The region at $1261 \mathrm{~cm}^{-1}-1194 \mathrm{~cm}^{-1}$ corresponds to breathing vibrations of the Pyrrole ring. The peak at $1294 \mathrm{~cm}^{-1}$ attributed to C-N in-plane deformation vibration mode. The bond of $\mathrm{C}-\mathrm{H}$ in-plane deformation vibration is situated at $1046 \mathrm{~cm}^{-1}$ and of the $\mathrm{C}-\mathrm{C}$ out of plane ring deformation vibrations or C-H rocking is at $681 \mathrm{~cm}^{-1}$. The C-H out-of-plane deformation vibration band of the ring has a peak at $924 \mathrm{~cm}^{-1}$. The presence of $801 \mathrm{~cm}^{-1}$ band conforms that predominantly $\alpha-\alpha$ ' bonding exists.

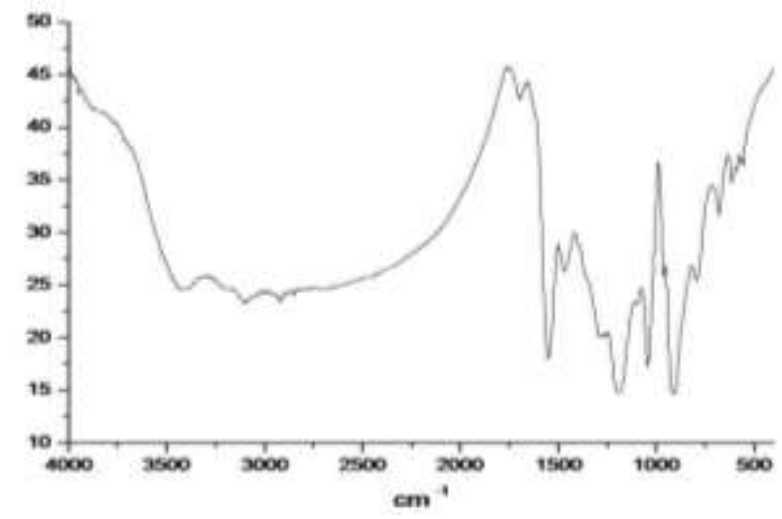

Fig.1 FTIR pattern for polypyrrole prepared using APS as oxidant

\section{$X$-ray diffraction analysis:}

A typical X-ray diffraction pattern for polypyrrole prepared using APS as an oxidant is shown in Fig.2.The XRD pattern shows broad peaks in the region $15^{0}<2 \square<30^{0}$ revealing that the resulting polypyrrole powders are amorphous. This agrees well with the structure reported in the literature. The broad peak usually indicates a short-range arrangement of chains. The half-width is measured from the diffraction curve from the angle higher than the peak position and assuming Gaussian distribution for the low angle part of the diffraction curve. 


\section{International Journal of Current Science Research and Review}

ISSN: 2581-8341

Volume 04 Issue 06 June 2021

DOI: 10.47191/ijcsrr/V4-i6-13, Impact Factor: 5.825

IJCSRR@ 2021

Www.ijesrr.org

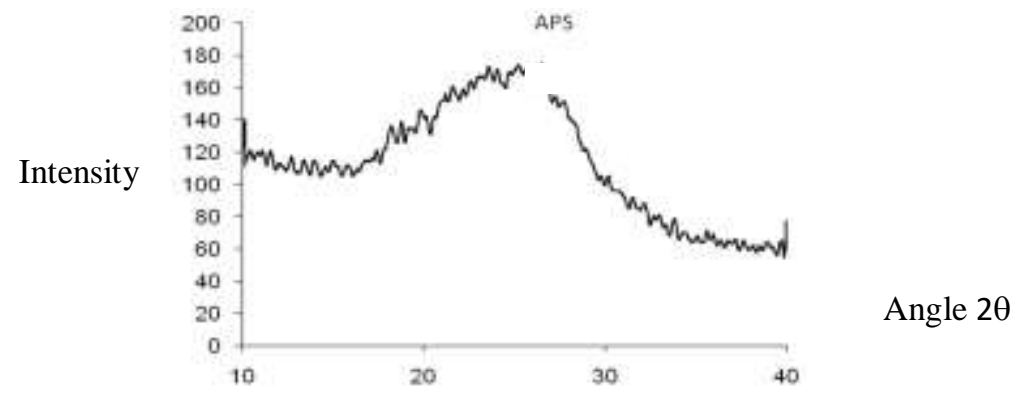

Fig. 2 X-ray diffraction pattern for polypyrrole prepared using APS as oxidant

\section{Morphology:}

The morphological features of polypyrrole synthesized chemically have revealed that mostly the growth is in the globular form. A typical SEM image is shown in Fig.3. The micrograph shows a globular structure. It can be seen from Fig. that when polymerization was done with APS the average size of globules was found to be $0.59 \square \mathrm{m} \square \square$ The sizes of these spherulites are varying from $0.2 \square \mathrm{m}$ to $0.7 \square \mathrm{m}$

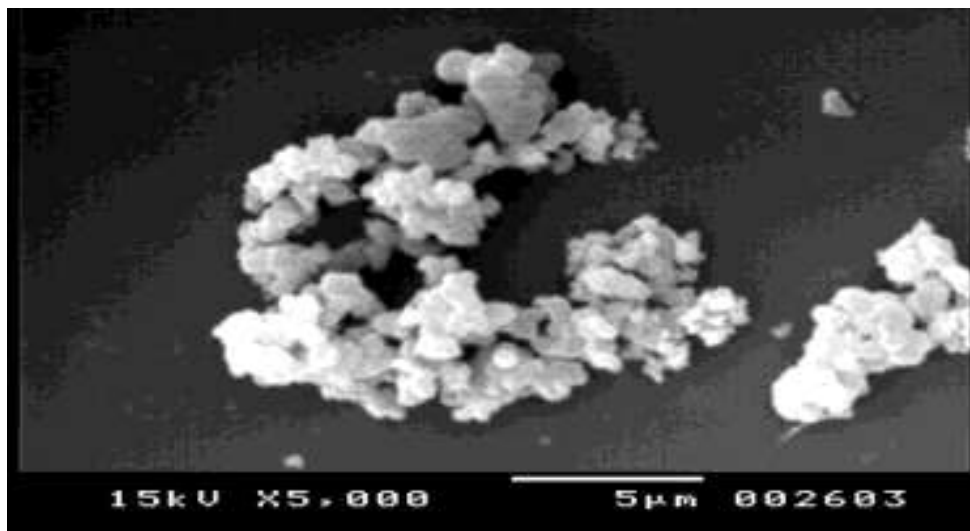

Fig. 3 SEM of PPy prepared by our method using APS as an oxidant

\section{I-V characteristics:}

Typical plots of I vs. V shows a nearly linear relationship of the graph of the I vs V curve was noted up to 8 volts as in Fig. 4. After this peak when voltage was increased further, a decrease in current was observed. The reason for such decrease is either that the current is now opposed by the flow of opposite charges in the opposite direction of degradation of the sample. Additional evidence for the contribution to the current by ions is being explored. The conductivities were found in the range of 1.0 to $4.6 \times 10^{-}$ ${ }^{3} \mathrm{~S} / \mathrm{cm}$.

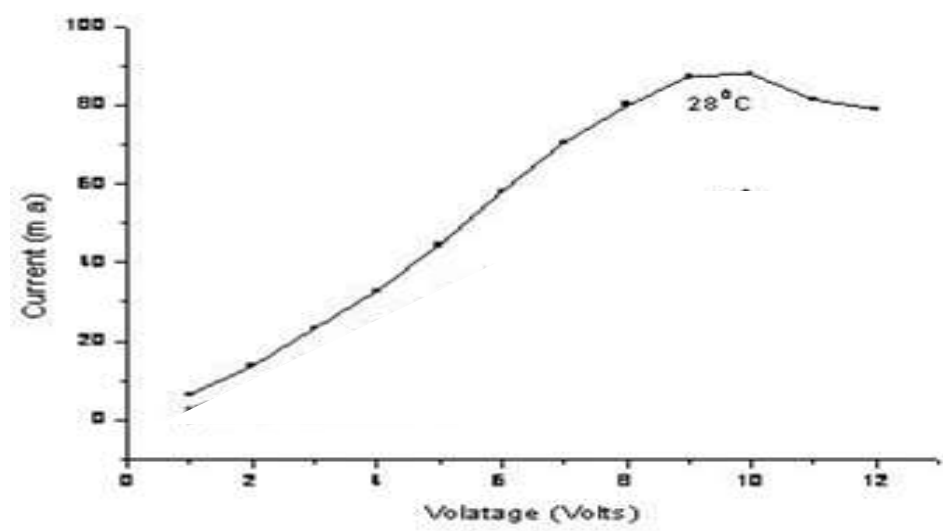

Fig. 4 I vs V graph of PPy with APS as oxidant 


\section{International Journal of Current Science Research and Review}

ISSN: 2581-8341

Volume 04 Issue 06 June 2021

DOI: 10.47191/ijcsrr/V4-i6-13, Impact Factor: 5.825

IJCSRR@ 2021

Www.ijcsrr.org

Film deposition in Plasma polymerization:

Polypyrrole was prepared in a plasma glass reactor (Fig. 5) specially designed to carry out R.F. plasma polymerizations. In a typical experiment, after several purging cycles with inert gas (12) (nitrogen), Pyrrole vapors were continuously allowed to enter from the monomer flask (6), into the cylindrical-shaped vacuum plasma reactor (1). The working pressure in the reactor due to monomer vapours was established to $0.2 \mathrm{~mm} \mathrm{Hg}$ and then the R.F. power was switched on. The power was transferred to the reactor through the semi-cylindrical, external, silver-coated electrodes (8). The R.F. power generator was capable of generating up to 250 $\mathrm{W}$. The polymer formed in the reactor was allowed to deposit on a glass plate ( Size 15 X $100 \mathrm{~mm}$ ), ITO plate, copper foils, and $\mathrm{KBr}$ window (9).

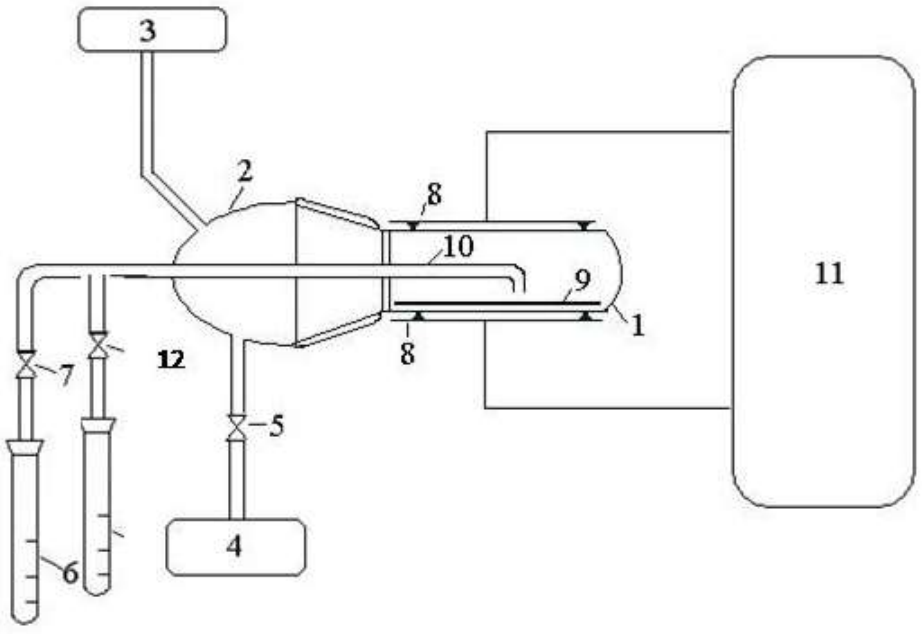

Fig. 5. Cold plasma installation for the Pyrrole polymerization:

1 - the cylindrical-shaped vacuum plasma reactor (neutral Pyrex glass, $\mathrm{L}=30 \mathrm{~cm}$, diameter $=4 \mathrm{~cm}$ ); 2 - close glass vacuum system; 3 - vacuum gauge Penning Pirani; 4 - vacuum pump; 5 and 7 - glass valves; 6 - monomer flask; 8 - semi-cylindrical external silver-coated electrodes; 9 - glass plate $(15$ X $100 \mathrm{~mm}) ; 10$ - central monomer's admission glass tube; 11 - R. F. generator (250 W, 13.6 Mhz). 12- Nitrogen cylinder

Before deposition, the reactor was evacuated with rotary pumps to a base pressure down to $2 \mathrm{~Pa}$. The precursor flow could be varied from 0.5 to $2.5 \mathrm{~g} / \mathrm{h}$, leading to a maximum pressure of $15 \mathrm{~Pa}$ during deposition. The deposition time was in the range $04-16$ min. The plasma polymerized Pyrrole films were placed in a Petri dish for $24 \mathrm{hrs}$. By this procedure, it is supposed to introduce charge carriers into the material which render it more conductive.

Thin, well adherent yellow-brown colour polypyrrole films deposited on the internal surface of the reactor and on the glass plates were obtained during the plasma polymerization in the gaseous phase of Pyrrole using a cylindrical glass reactor (Fig. 5). The film thickness could be controlled using different times of glow discharge in the reactor. For short discharge times, adherent films were obtained on metallic or glass supports. Most of the polymer obtained was insoluble in organic solvents.

The FT-IR spectra of plasma polymerized PPy film is presented in Fig. 6. These show several common absorption bands, the most important ones are related to the $\mathrm{C}-\mathrm{H}$ out of plane deformation vibration in the ring, in the region $700-730 \mathrm{~cm}^{-1}$, there are three well-resolved bands in the region of $1550 \mathrm{~cm}^{-1}$ to $1700 \mathrm{~cm}^{-1}$, of which the one at $1670 \mathrm{~cm}^{-1}$ can be assigned to formation $\mathrm{C}=\mathrm{O}$. Although PPy does not contain any oxygen it may be getting reacted from the air or moisture. This fact reveals that the PPy ring is getting decomposed to some extent and may appear as an aliphatic component in the polymerization process. This is likely to weaken the ring-to-ring bonding of PPy which is seen to be undisturbed in chemical or electrochemical polymerization. Therefore it will affect electrical conductivity. 


\section{International Journal of Current Science Research and Review}

ISSN: 2581-8341

Volume 04 Issue 06 June 2021

DOI: 10.47191/ijcsrr/V4-i6-13, Impact Factor: 5.825

IJCSRR@ 2021

www.ijjcsrr.org

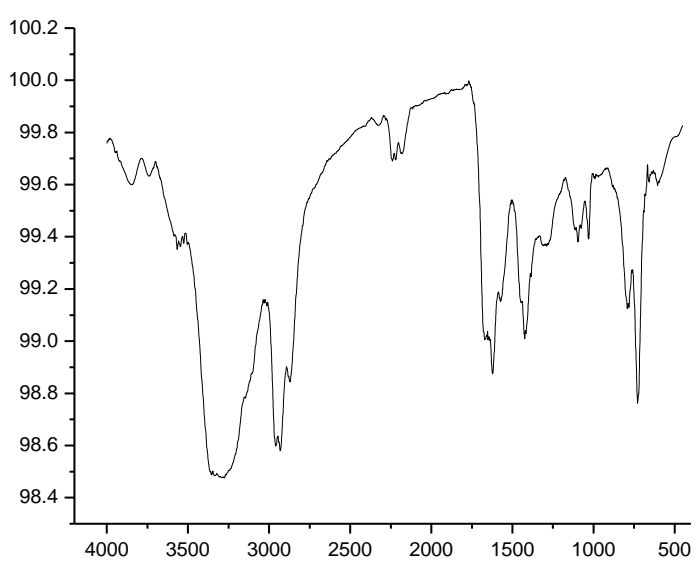

Fig 6. FTIR Spectra for plasma polymerization of PPy

\section{Morphology:}

The morphology of the plasma polymerized Pyrrole films grown on ITO glass plates was observed by SEM. Fig. 7 shows that the film grows in the form of globular structure, the average size of globules being 1 micron. The changes in the morphology can be due to the diffusion of dopant ions into the films. Therefore changes in some other characteristics may also be expected.

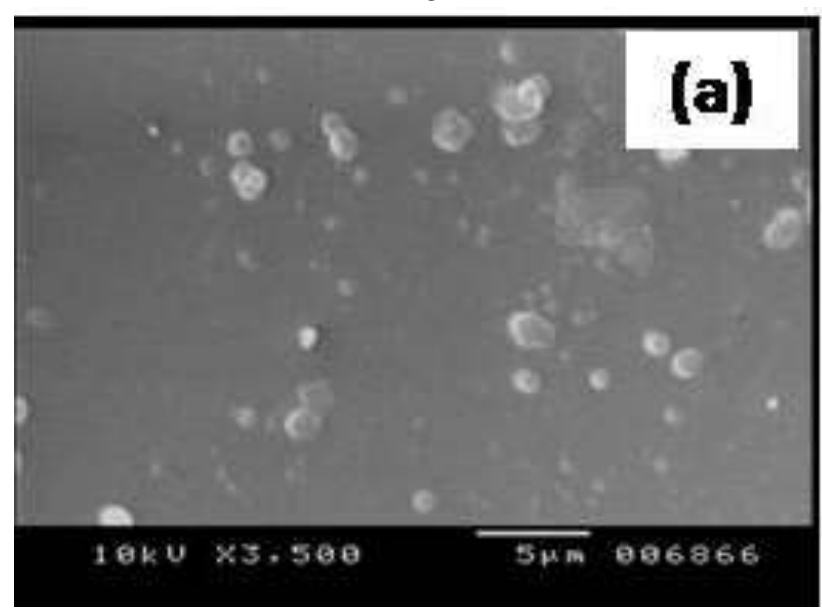

Fig. 6 SEM of Plasma Controlled

\section{I-V characteristics:}

The I-V characteristic of plasma polymerized PPy thin film is shown in Fig. 8 It shows a linear relationship showing ohmic-like behaviour. The value of conductivity calculated from the slope of this graph is $9.3 \times 10^{-3}$. The low value indicates that in PPy the bonding is not favourable for the conduction process. This result is supported by the FTIR discussion where it is pointed out that the ring opens and some aliphatic groups bond with the PPy rings. This will lead to cross liking and reduction in the electrical conductivity.

The study of the I-V characteristic for the doped samples surprisingly does not show any enhancement in the conductivity, although some changes in the morphology were noted. 


\section{International Journal of Current Science Research and Review}

ISSN: 2581-8341

Volume 04 Issue 06 June 2021

DOI: 10.47191/ijcsrr/V4-i6-13, Impact Factor: 5.825

IJCSRR@ 2021

www.ijcsrr.org

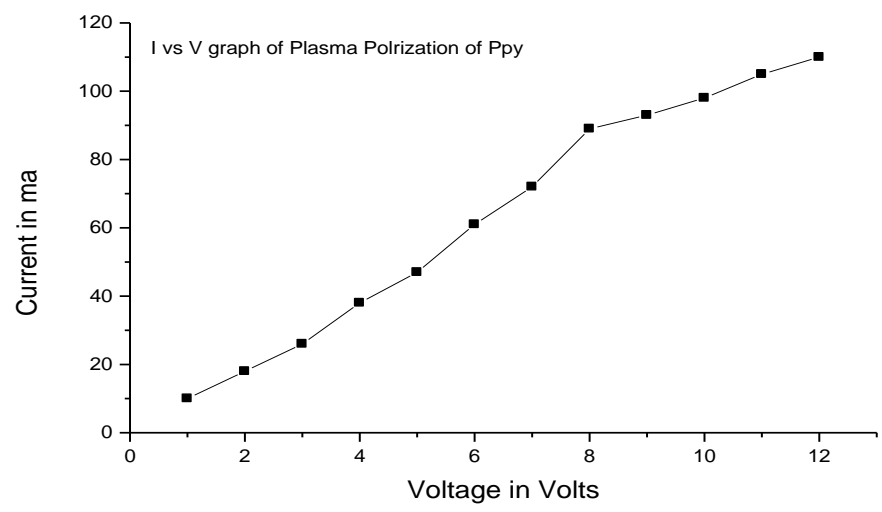

\section{CONCLUSIONS}

Polymerization of Pyrrole using various methods like chemical oxidative and Plasma irradiation have been investigated. The main purpose of using different routes was to study the mechanisms involved in each method and evaluate the advantages of each method.

In Plasma Polymerization, it is possible to modify the surface properties of a substrate, while retaining the transparency and bulk properties of the substrate material or to deposit thin transparent films. Furthermore, it is a solvent-free, fast and versatile process.

\section{REFERENCES}

1. P. J. Nigrey, A. G. Mac Diarmid and A. J. Heeger, J. Chem. Soc. Chem. Commu. 594 (1979)

2. W. Deits, P. Cukor, M. J. Jonson, Synth Met. 4, 189 (1982)

3. F. Diaz, K. K. Kanazawaand G,. P. Gardian, J. Chem. Soc. Commu. 635 (1979)

4. Wang Lin-Xia, Lia Xin-Gui, and Yang Yu-Liang. 2001. Reactive and Functional Polymers. 47:125.

5. da Cruz, A.G.B., J.L. Wardell, and A.M. Rocco. 2006. Synth. Met.

6. Elsenbaumer, R.L. and J. R. Reynolds. 1997. Handbook of Conducting Polymers. T.A. Skotheim (ed.). Marcel Dekker: New York, NY. Volumes 1-2.

7. Hemant K. Chitte, Narendra V. Bhat,, Vasant E. Walunj, Ganesh N. Shinde, Journal of Sensor Technology, 2011, Volumes $1,47-56$

8. Zhang et al., J Am Chem Soc 2004, 126, 6572.

9. Hsueh, C.; Brajter-Toth, A. AnaL Chem. 1994, 66, 2458-2464.

10. Garcia, J.; Quintela, P. A.; Kaifer, A. E. AnaL Chem. 1989,61,979-981.

11. Chastel, O.; Kauffmann, J. M.; Patriarche, G. J. AnaL Chem. 1989,61, 170-173.

12. Hemant K. Chitte, Narendra V. Bhat, Ganesh N. Shinde ,Materials Sciences and Applications, 2011, 2, 1491-1498

13. Tanaka, K.; Tamamushi, R. J. ElectroanaL Chem. 1987,236,305-307.

14. Noftle, R. E.; Pletcher, D. J. Eleetrochem. Chem. 1990,293, 273-2n.

15. MusSini, T.; Faita, G. In Encyclopedia ofElecrrochemistry ofthe Elements; A. J.

16. Marian TOTOLIN* and Mircea Grigoras Plasma Induced PPy Polymerization Revue Roumaine de Chimie, 2007, 52(10), 999-1005

17. Y. Xie, Z. Qiao, M. Chen, X. Liu, Y. Qian, Adv. Mater. 11 (1999) 321.

18. S. Milojković, D. Kostoski, J. Čomor, J.M. Nedeljković, Polymer 38 (1997) 2853.

19. T. Seguchi, T. Yagi, S. Ishikawa, Y. Sano, Radiat. Phys. Chem. 63 (2002) 35.

20. X. Zhang, M. Wang, T. Wu, S. Jiang, Z. Wang, J. Am. Chem. Soc. 126, (2004) 6572.

Cite this Article: Dr. Chitte Hemant Keshav(2021). Comparative Study of Synthesis of Polypyrrole Using Chemical Polymerization Technique and Plasmapolymerization. International Journal of Current Science Research and Review, 4(6), $578-583$ 